\title{
Highly Multiplex Targeted Proteomics Enabled by Real-Time Chromatographic Alignment
}

\section{Supplementary information}

\author{
Philip M. Remes ${ }^{*}$, Ping Yip ${ }^{1}$, Michael J. MacCoss ${ }^{2 *}$ \\ ${ }^{1}$ Thermo Fisher Scientific, 355 River Oaks Parkway, San Jose, California 95134 \\ philip.remes@thermofisher.com; maccoss@uw.edu
}

${ }^{2}$ Department of Genome Sciences, University of Washington, $372015^{\text {th }}$ St. NE, Box 355065, Foege Building S113D, Seattle WA 98195

\section{Table of Contents}

1. Research mass spectrometer system

2. Cross correlation of mass spectra

3. Quality filters

4. MS1-only based cross-correlation

5. Human plasma dilution in chicken plasma

6. Coefficient of variation versus retention time

7. Duty cycle

\section{Research mass spectrometer system}

The mass spectrometer system used for these studies is a modified version of the Orbitrap Fusion Lumos ${ }^{\mathrm{TM}}$. The linear ion trap mass analyzer in this system is capable of scanning at about $65 \mathrm{~Hz}$ for a mass range of m/z 200-1500, which is somewhat faster than production Lumos or Eclipse systems (closer to $45 \mathrm{~Hz}$ ). The $7 \mathrm{~ms}$ difference in scan periods is due to several factors. On the one hand, the research system uses a somewhat higher pressure of Helium gas in the linear ion trap. This additional gas allows to reduce the length of the transfer time from high to low pressure linear ion trap cells, at the cost of a modest reduction in mass resolution. Additionally, the research and Eclipse systems have a version of quadrupole DC rod drivers that have a much faster slew rate than previous instrument generations, allowing to shave off 1-2 ms of overhead for the event where ions travel from the source all the way through the quadrupole to the gate. The Eclipse does not yet make full use of this hardware improvement, in part because this event is also limited by the time of flight of the ions, and because production Tribrid instruments are subject to more strict constraints on ion transfer times due to the wide variety of compounds and experiments that customers may use, and we must provide a set of parameters that will ensure good performance for instruments produced on a production scale. In the research environment, instrument developers can sometimes tune various parameters to the very edge in a way that is not yet suitable for robust distribution to the customer.

Currently, a Lumos production system is capable of a linear ion trap mass analysis rate of $43 \mathrm{~Hz}$ for the same $1250 \mathrm{Th}$ scan range used in this paper, while the research instrument in these studies scanned at $65 \mathrm{~Hz}$. Since the number of targets that can be assayed in a given time will scale linearly with the acquisition rate (i.e. as $\frac{43}{65}=0.662$ ), a production system would have capacity for 21 DIA alignment scans in $0.5 \mathrm{~s}$, and be capable of analyzing 985 or 3872 targets for the conditions described above.

The linear ion trap mass analyzer was used for these studies, although a Lumos system also has an Orbitrap mass analyzer that can acquire spectra at a rate of about $24 \mathrm{~Hz}$ with the $7.5 \mathrm{k}$ resolution setting when injecting ions for the same $13 \mathrm{~ms}$ used with the linear ion trap. Using the same math as the preceding paragraph, the cognate Orbitrap PRM experiment would use 11 DIA scans and 549 or 2215 targets. We leave any comparative analysis of the analytical merits of the two analyzers for future studies; however, the principle themes would likely be the relative importance of the speed and single ion detection limits of the linear ion trap compared to the high resolution and accurate mass of the Orbitrap. We note however that peptide analysis is perhaps the ideal mass spectrometry 
application for a nominal mass analyzer: due to the large number of possible transitions, there is a high probability that at least 5 of them are free from interferences. ${ }^{1}$

We have also performed our alignment procedure with Orbitrap MS1 spectra ${ }^{1}$. Our current implementation discards any possible advantage conferred by the mass accuracy and resolution with the memory-saving compression step. If the alignment procedure was performed on a computer with more memory and computing power than the instrument's embedded processor, perhaps these analyzer capabilities could be better exploited.

\section{Cross correlation of mass spectra}

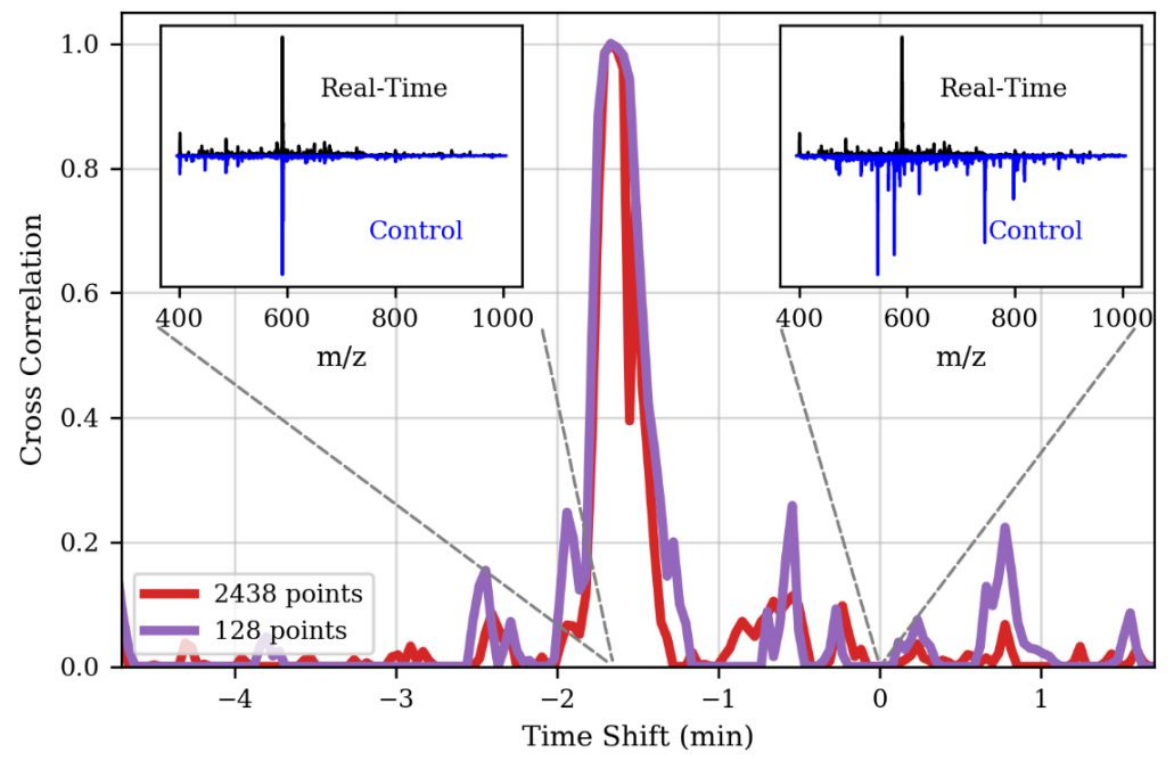

Figure S1. Example cross-correlation for MS1 spectra for m/z 350-1005, binned to 2439 points with 0.250125 Da spacing (red), compared to the same spectra Haar compressed to 128 points (purple). The insets show the real-time mass spectra on the positive y axis in black compared to the control spectra on the negative y axis in blue.

\section{Quality Filters}

The quality filters used for retention time shift estimations included the following requirements summarized in Equation S1: the number of peaks in the cross-correlation $\eta_{\alpha}$ with area larger than a threshold $f_{\alpha}$ should be 1 ; the width of the largest peak $\omega$ should be less than a threshold $f_{\omega}$; the difference between the peak position on this iteration and the last iteration should be less than a threshold $f_{\delta}=10 \mathrm{~s}$. If any of these requirements are not met, the shift from the last cycle $\delta_{t-1}$ is maintained for the new cycle $t$. The values of the filters $f_{\omega}$ and $f_{\alpha}$ are determined empirically from analysis of the auto correlations in the control data set. If all the quality filters are passed, then the new shift value is stored, after passing it through an exponential filter $G$ with a characteristic time constant of 30 seconds.

$$
\delta_{t}=\left\{\begin{array}{c}
\eta_{\alpha} \neq 1 \rightarrow \delta_{t-1} \\
\omega>f_{\omega} \rightarrow \delta_{t-1} \\
\left|\delta_{t}-\delta_{t-1}\right|>f_{\delta} \rightarrow \delta_{t-1} \\
e l s e \rightarrow G\left(\delta_{t}\right)
\end{array}\right.
$$

We have considered the use of a "latch", that would ensure that once a target was placed on the active list of targets, it would remain for a certain length of time. Such a procedure could keep a target from bouncing in and off the active list near the boundaries of its 
acquisition segment. We plan to explore this technique in the future, but at present the results do not seem to suffer from this effect, likely because of the quality filters described above.

\section{MS1-only based cross-correlations}

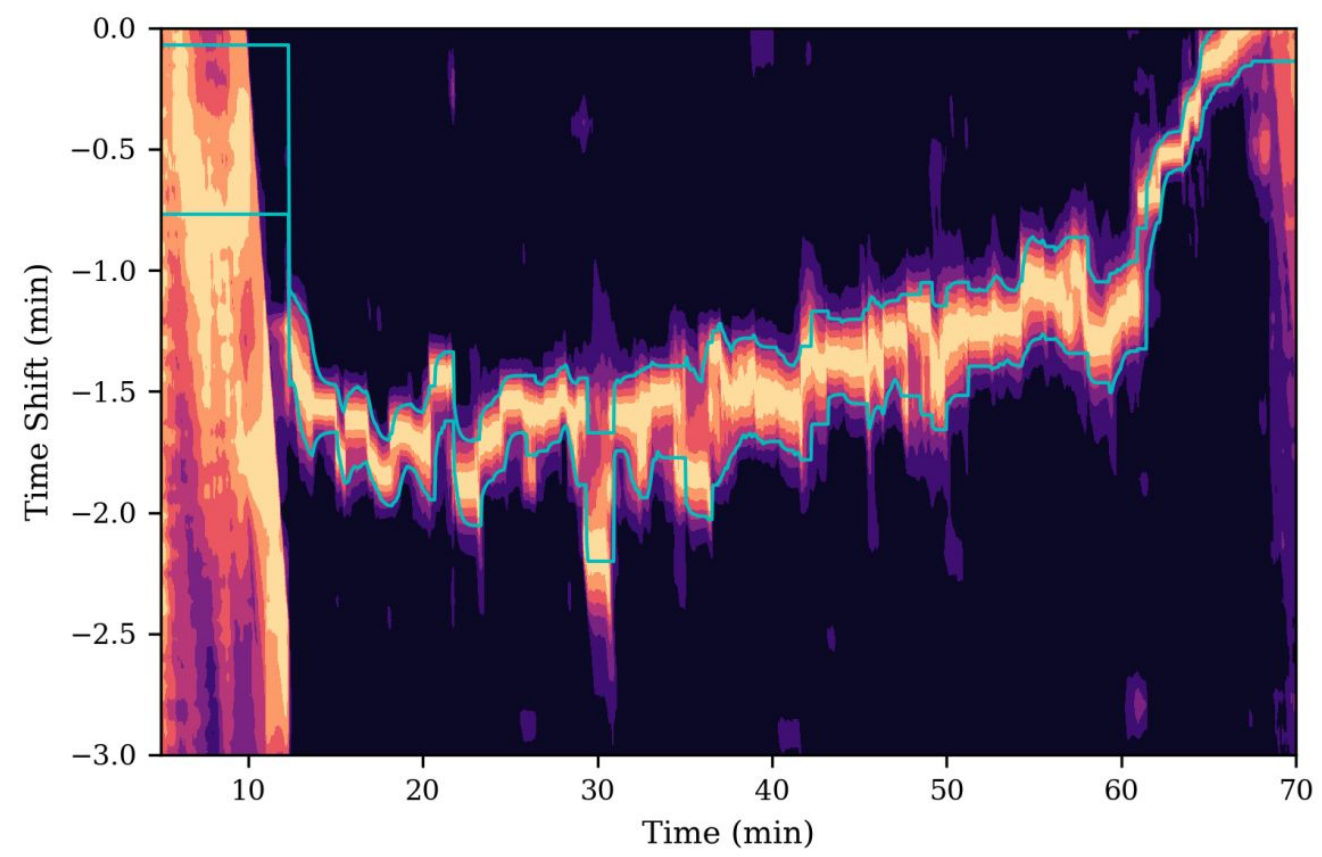

Figure S2. Cross-correlations versus time for a targeted assay aligned to a control data set acquired 16 days prior, using MS1 spectra only. These data are from the exact same experiment as Figure 2 in the text, where only the MS1 spectra were used for alignment. Lighter colors denote high similarity to the control, while dark regions denote low similarity. The cyan lines denote boundary positions of the apex peak in the cross-correlation. Compared to the case of using DIA MS/MS spectra for alignment, there occasionally are segments of time where the estimated retention time shift is incorrect (ex. 20 and 30 minutes). 


\section{Human Plasma Dilution with Chicken Plasma}

In the course of performing preliminary sensitivity experiments, we assayed peptides in human plasma at several different dilutions in chicken plasma, using MS1-only scans for chromatogram alignment. Doing this study, we realized that the human-only reference data set was able to perform alignment when only $6 \%$ of the human peptides were present in the sample matrix diluted with chicken plasma. Below a $6 \%$ dilution, the alignment was sometimes poor and noisy, examples of which can be appreciated in Figure S3b. The preliminary conclusion is that the human plasma peptide signatures are quite unique, and the $\mathrm{S} / \mathrm{N}$ of the cross-correlations at the $6 \%$ dilution level are sufficient to align to across much of the LC gradient. Cross-correlations are regarded for their ability to perform this kind of signal extraction in noisy backgrounds and have been used for decades in applications such as radar and satellite communications. These results bode well for the future application of our technique to samples from different cell lines, patient samples, and disease states, etc."
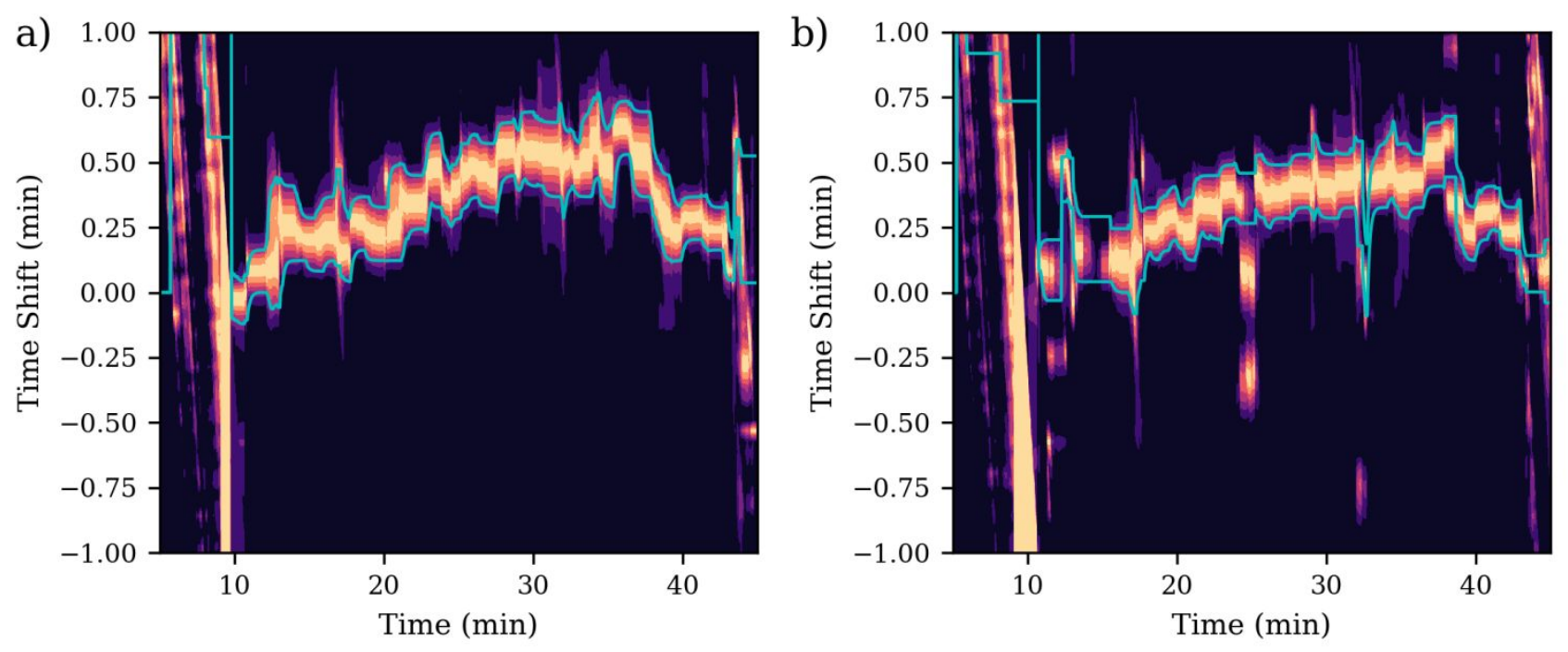

Figure S3. Retention time alignment example using dilutions of human with chicken plasma. The control data were MS1 spectra compressed to 512 points. Alignment was performed against a) the same $100 \%$ human sample 5 days after the control data were acquired b) a $6 \% / 94 \%$ human/chicken dilution.

\section{Coefficient of Variation vs Retention Time}

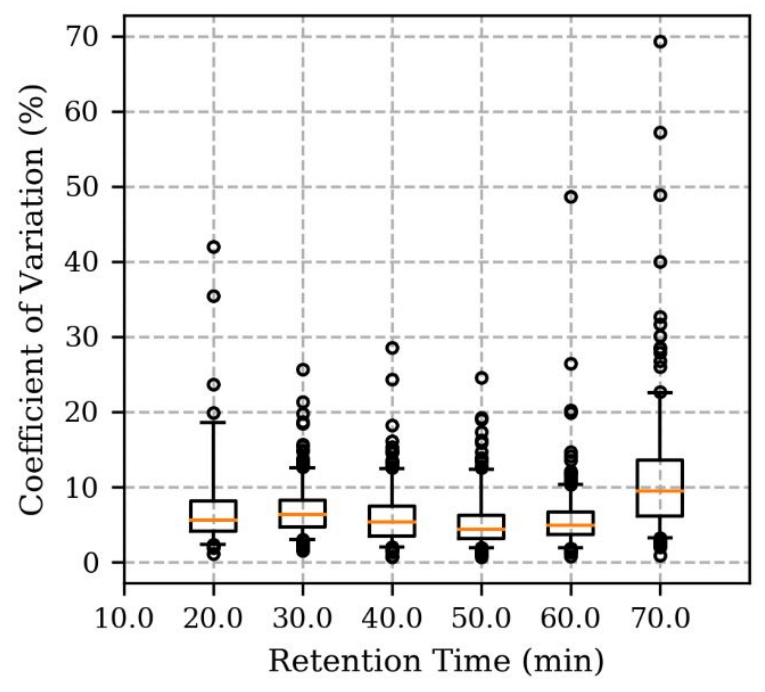


Figure S4. Box and whisker plots for CV of 5 replicates separated by retention time.

\section{Duty Cycle}

Another way of looking at the efficiency of instrument usage is to compute an experimental duty cycle $D$, which denotes the fraction of time the instrument is used to perform a scan for an analyte during an acquisition segment, which is plotted below. This is computed as in Equation 5, where $A$ is the number of active targets, $t_{\text {peak }}$ is the LC peak width, and $t_{\text {seg }}$ is the acquisition segment duration.

$$
D=\frac{1 t_{\text {peak }}}{A t_{\text {seg }}}
$$

This kind of duty cycle is distinct from the intra-scan level duty cycle that is often a major consideration in the design and operation of a mass spectrometry ${ }^{2}$. Instead, the experiment duty cycle is a measure of how well instrument resources are being allocated to the measurement of a set of analytes. As acquisition segment is decreased, there are fewer active targets per cycle, and also fewer scans are wasted at times where there is no analyte. Together this makes the duty cycle increase as the square of the reduction in segment duration. This means that if the experimentalist could "choose" to distribute analyte elution times in an optimum way, the instrument time available to analyze each one would increase as the square of segment duration. However, for a fixed distribution of targets, smaller segments provide for linearly more scan time per precursor.

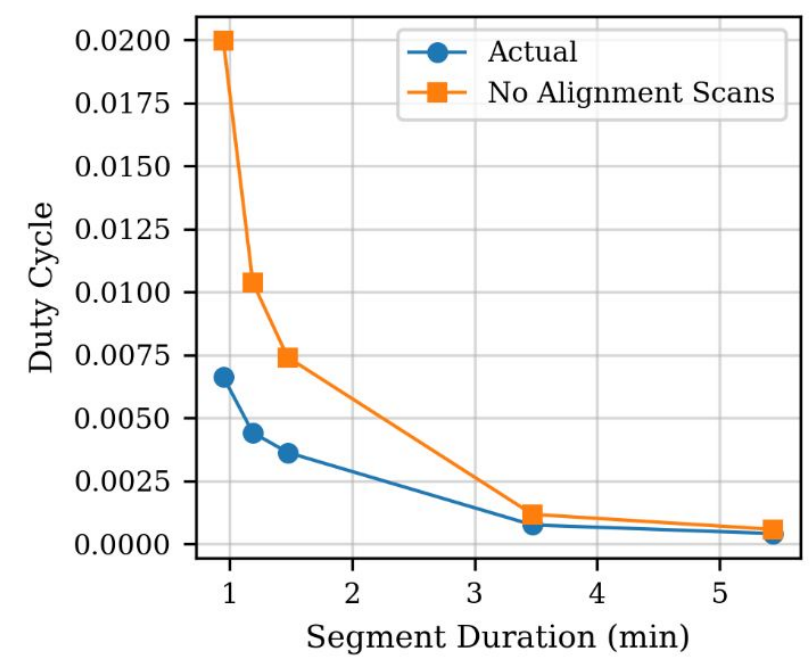

Figure S5. Median experiment duty cycle, which is the fraction of time that scans are used for a given analyte during an acquisition segment

(1) Remes, P. M.; Yip, P.; Huguet, R. Retention Time Correction Method Utilizing Unspecified Peaks in MS Scans. ASMS Annual Conference on Mass Spectrometry and Allied Topics. Atlanta, GA., 2019.

(2) Senko, M. W.; Remes, P. M.; Canterbury, J. D.; Mathur, R.; Song, Q.; Eliuk, S. M.; Mullen, C.; Earley, L.; Hardman, M.; Blethrow, J. D.; Bui, H.; Specht, A.; Lange, O.; Denisov, E.; Makarov, A.; Horning, S.; Zabrouskov, V. Novel Parallelized Quadrupole/Linear Ion Trap/Orbitrap Tribrid Mass Spectrometer Improving Proteome Coverage and Peptide Identification Rates. Anal. Chem. 2013, 85 (24), 11710-11714. https://doi.org/10.1021/ac403115c. 